\title{
(6) OPEN ACCESS \\ Cause-specific neonatal mortality: analysis of 3772 neonatal deaths in Nepal, Bangladesh, Malawi and India
}

\author{
Edward Fottrell, ${ }^{1}$ David Osrin, ${ }^{1}$ Glyn Alcock, ${ }^{1}$ Kishwar Azad, ${ }^{2}$ Ujwala Bapat, ${ }^{3}$ \\ James Beard, ${ }^{1}$ Austin Bondo, ${ }^{4}$ Tim Colbourn, ${ }^{1}$ Sushmita Das, ${ }^{3}$ Carina King, ${ }^{1}$ \\ Dharma Manandhar, ${ }^{5}$ Sunil Manandhar, ${ }^{5}$ Joanna Morrison, ${ }^{1}$ Charles Mwansambo, ${ }^{6}$ \\ Nirmala Nair, ${ }^{7}$ Bejoy Nambiar, ${ }^{1}$ Melissa Neuman, ${ }^{1}$ Tambosi Phiri, ${ }^{8}$ Naomi Saville, ${ }^{1}$ \\ Aman Sen, ${ }^{5}$ Nadine Seward, ${ }^{1}$ Neena Shah Moore, ${ }^{3}$ Bhim Prasad Shrestha, ${ }^{5}$ \\ Bright Singini, ${ }^{8}$ Kirti Man Tumbahangphe, ${ }^{5}$ Anthony Costello, ${ }^{1}$ Audrey Prost ${ }^{1}$
}

\section{- Additional material is published online only. To view please visit the journal online (http://dx.doi.org/10.1136/ archdischild-2014-307636). \\ For numbered affiliations see end of article. \\ Correspondence to Dr Edward Fottrell, University College London, Institute for Global Health, 30 Guilford Street, London WC1N 1EH, UK; e.fottrell@ucl.ac.uk}

Received 2 October 2014 Revised 13 April 2015 Accepted 14 April 2015 Published Online First 13 May 2015

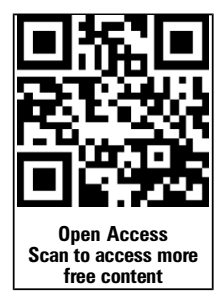

\section{SLinked}

- http://dx.doi.org/10.1136/ fetalneonatal-2015-308822

\section{ABSTRACT}

Objective Understanding the causes of death is key to tackling the burden of three million annual neonatal deaths. Resource-poor settings lack effective vital registration systems for births, deaths and causes of death. We set out to describe cause-specific neonatal mortality in rural areas of Malawi, Bangladesh, Nepal and rural and urban India using verbal autopsy (VA) data.

Design We prospectively recorded births, neonatal deaths and stillbirths in seven population surveillance sites. VAs were carried out to ascertain cause of death. We applied descriptive epidemiological techniques and the InterVA method to characterise the burden, timing and causes of neonatal mortality at each site.

Results Analysis included 3772 neonatal deaths and 3256 stillbirths. Between $63 \%$ and $82 \%$ of neonatal deaths occurred in the first week of life, and males were more likely to die than females. Prematurity, birth asphyxia and infections accounted for most neonatal deaths, but important subnational and regional differences were observed. More than one-third of deaths in urban India were attributed to asphyxia, making it the leading cause of death in this setting. Conclusions Population-based VA methods can fill information gaps on the burden and causes of neonatal mortality in resource-poor and data-poor settings. Local data should be used to inform and monitor the implementation of interventions to improve newborn health. High rates of home births demand a particular focus on community interventions to improve hygienic delivery and essential newborn care.

\section{INTRODUCTION}

Each year, approximately three million children die in the first 28 days after birth, predominantly due to complications of preterm birth, asphyxia and sepsis. ${ }^{1-3}$ With postneonatal mortality declining faster than neonatal mortality, ${ }^{4}$ these deaths account for a growing proportion of under-five deaths. Understanding the numbers and causes of neonatal deaths, as well as gender differences and national and subnational variation, is key to realising the Every Newborn Action Plan and post-Millennium Development Goals of a 'grand

\section{What is already known on this topic?}

Globally, there are three million neonatal deaths every year, mostly from prematurity, asphyxia and sepsis.

- Ninety-eight per cent of these deaths occur in low-income countries, where poor mortality data hinders intervention strategies.

- More direct measurement could guide policy and practice at national and subnational levels.

\section{What this study adds?}

Population-based verbal autopsy (VA) tools such as InterVA offer a standardised method to directly measure the burden and causes of neonatal mortality in low-income settings.

- Although patterns of neonatal death differ between sites, both early and late neonatal mortality remain unacceptably high.

- Localised, direct measurement reveals important subnational variations in mortality rates and causes, which might be masked by estimation methods at the national level.

convergence' in health, with substantial reductions in neonatal and child mortality. ${ }^{5-8}$ Unfortunately, the resource-poor settings that bear the burden of more than $98 \%$ of neonatal deaths often lack the effective vital registration systems crucial to understanding mortality and planning services or interventions.

Advances in epidemiological modelling methods have recently been applied to pooled datasets to characterise neonatal mortality. ${ }^{5}$ Such estimates are useful at a global level, but have limitations. Broad underlying assumptions, lack of transparency in the data and methods used and restrictions on disaggregating the data limit their relevance at subnational levels, where there can be substantial variation in rates, trends and cause distributions. There is a growing recognition that measurement rather than modelling is needed ${ }^{10}{ }^{11}$ and frustration at 
unsatisfactory progress with civil registration systems. Global bodies, including the WHO, call for the application of fit-for-purpose methods for registering deaths and assigning their causes in a consistent, systematic and timely manner. ${ }^{12-16}$

Since 2001, we have implemented community-based surveillance of perinatal events along with verbal autopsies (VA) in rural Nepal, Bangladesh, Malawi and urban and rural India. This has enabled us to prospectively document births, neonatal deaths and their causes within geographical areas covering a total population of approximately 2.4 million. Using these data, we describe the rates, timing and causes of neonatal mortality for 3772 deaths from low-income and middle-income settings, highlighting regional and gender disparities and identifying priorities for public health intervention.

\section{METHODS}

\section{Study populations}

We used data gathered between 2001 and 2011 in cluster randomised controlled trials of community mobilisation 'women's group' interventions in Bangladesh (Perinatal Care Project $(\mathrm{PCP})^{17}{ }^{18}$ ), Malawi (MaiMwana ${ }^{19}$ and MaiKhanda ${ }^{20}$ ), India (Society for Nutrition, Education and Health Action $(\mathrm{SNEHA})^{21}$ and Ekjut ${ }^{22}$ ) and Nepal (Makwanpur and Dhanusha $^{23}$ ). The data represent 118084 births recorded in seven locations with community-based surveillance of perinatal events. Characteristics of each study population, study timelines and basic neonatal health indicators are summarised in table 1. Given the documented effect of the women's group intervention on neonatal mortality, ${ }^{24}$ only data from counterfactual clusters without women's groups were included in the analysis. Counterfactual clusters were not pure control areas in every setting: in MaiMwana, Malawi, a breastfeeding counselling intervention was implemented and tested in 12 of the 24 counterfactual clusters; ${ }^{19}$ in PCP, Bangladesh, four of the nine clusters received training of traditional birth attendants on resuscitation; ${ }^{25}$ in Dhanusha, Nepal, a community-based neonatal sepsis management intervention was implemented in 9 of the 30 counterfactual clusters. $^{26}$

\section{Community-based surveillance and verbal autopsy}

Surveillance systems for births and newborn deaths were established in each setting. Their design and implementation have been described elsewhere. ${ }^{17} 202123 \quad 26-30$ Field-based key informants recruited in cluster subareas identified births and deaths, which were then verified by trained interviewers. At all sites, stillbirths were distinguished from very early neonatal deaths based on the absence of signs of life (movement, breathing, crying) when the infant was born, according to the mother or other caregivers. In MaiKhanda, Malawi, a simple algorithm was used to classify deaths as stillbirths or neonatal deaths from data pertaining to these reported signs of life. ${ }^{30}$

To ascertain likely causes of neonatal deaths, trained lay interviewers conducted VAs. VA is the process of interviewing a caregiver, relative or witness to ascertain the presence, absence or nature of signs, symptoms and circumstances observed at or around the time of death. ${ }^{31}$ Interviewers used a structured questionnaire to gather VA information, usually from the mother of the deceased child. Questionnaires comprised a series of fixed-response questions, with space to record open-ended comments, but this open-ended information was not included in the current analysis, as previous work has shown that it does not add to biomedical interpretations of cause of death using automated methods. ${ }^{32}$ The questionnaires were similar in all settings (see online supplementary table S1), and interviewers were trained to ask all relevant questions on the VA questionnaire, adhering to predefined skip patterns, regardless of perceived relevance to the specific case. Interviews were conducted after a culturally appropriate mourning period. Only VAs with sex and age at death data were considered to be complete and included in the study. The VA completion rates for each site are summarised in table 1 .

\section{Data management and quality control}

Registered vital events identified by incentivised key informants or salaried enumerators were confirmed by interviewer visits to households and, in a subsample of cases, through field supervisor visits. Trends were compared with local public system registration figures. Further quality checks during in-country electronic data entry mandated referral back to the field for correction when errors were detected. ${ }^{17-23} 26-30$

\section{Interpreting verbal autopsy}

VA data were interpreted through InterVA V.4.02 (http://www. interva.net), an automated method for interpreting signs, symptoms and circumstances. ${ }^{33}$ Based on the reported presence or absence of indicators, Bayesian reasoning is applied to calculate the likelihood of 60 possible cause-of-death categories compatible with the International Classification of Diseases V.10. Following procedures outlined in the InterVA user guide, ${ }^{34}$ variables describing specific signs and symptoms were mapped to equivalent InterVA indicators to generate files to be processed through the Bayesian model. For example, any positive responses to questions about intermittent or fast breathing, chest in-drawing, grunting or nasal flaring before death were used to generate a positive response in InterVA's 'difficulty breathing' indicator. One member of the research team (EF) reviewed the mapping between each study site's data and the InterVA input file as a quality control measure to check plausibility of symptom frequencies.

InterVA requires population HIV and malaria prevalence to be specified so that the model can account for baseline differences between locations. Previous research has shown that simple descriptions of 'very low', 'low' or 'high' prevalence, corresponding to increasing orders of magnitude, are adequate for this. ${ }^{35}$ Malaria and HIV settings for each site are summarised in table 1.

InterVA reports the probability of up to three of the most likely causes for each death. ${ }^{33}$ We summed the likelihoods of each cause from every individual death to estimate the burden of each at population level. Dividing this estimate by the total number of deaths provided population cause-specific mortality fractions (CSMFs), and dividing by the number of live births at each study site provided estimates of cause-specific mortality rates (CSMRs). In MaiMwana (Malawi) and all Asian sites, it was possible to calculate mortality rates by sex, based on the directly measured number of liveborn males and females. In MaiKhanda (Malawi), however, sex was not recorded for all live births, and estimates of the numbers of liveborn males and females were derived by splitting the total number on the basis of the 2010 Demographic and Health Survey live-birth sex ratio for 2007-2010. ${ }^{36}$

\section{Ethical considerations}

The trials in which the current data were gathered were approved by either the University College London or the Great Ormond Street Hospital Research Ethics Committee in the UK, and by ethical review committees in each setting: the Ethical Review Committee of the Diabetic Association of Bangladesh, 
the Nepal Health Research Council (with a Memorandum of Understanding with the Government of Nepal Ministry of Health), the Independent Ethics Committee for Research on Human Subjects (Mumbai), an independent ethical committee in Jamshedpur, India, and the Malawi National Health Sciences Research Committee.

\section{RESULTS}

A total of 3772 neonatal deaths with complete age and sex data were included in the analysis. The average time from death to VA interview varied between study sites, with an average of 3 weeks at Ekjut and SNEHA (India), 5-6 weeks at PCP (Bangladesh) and Dhanusha (Nepal), 29 weeks at MaiMwana and 67 weeks at MaiKhanda (Malawi). The relatively long death-to-interview time in MaiKhanda reflects the fact that a large number of VAs were not completed initially, but rather included as part of a verification exercise after the trial ended in May 2011. ${ }^{30}$ Interview dates were not available for Makwanpur (Nepal). The average number of VA indicators per case was 12 for MaiKhanda, 13 for Makwanpur, 14 for Dhanusha, Ekjut and MaiMwana, 15 for SNEHA and 17 for PCP. Numbers of stillbirths and neonatal deaths contributed by each study site are presented in table 2, which also shows crude and sex-specific mortality rates and mortality rate ratios comparing males with females.

Table 3 shows sex-specific and cause-specific neonatal mortality fractions for all InterVA-derived cause categories. Infectious causes-diarrhoeal disease, meningitis and encephalitis, pneumonia and sepsis-were consolidated into a broad cause category of infection, and the categories 'other and unspecified' and 'indeterminate' were consolidated to facilitate presentation of cause-specific rates and overall fractions in figure 1.

Crude sex-specific neonatal mortality rates and rate ratios (table 2) show that males had 20\%-50\% higher mortality than females, with rate ratios (95\% CIs) ranging from 1.2 (1.0-1.3) in MaiKhanda to 1.5 (1.1-2.0) in Makwanpur. In most settings, there were no obvious important differences between cause distributions by sex (table 3). However, at SNEHA in urban India and, to a lesser extent, Makwanpur in Nepal, a larger proportion of female deaths were attributed to prematurity.

Figure 2 shows CSMFs by day of death. Across all Asian sites, approximately one-third of neonatal deaths occurred on the first day of life. This proportion was greater than $40 \%$ in both Malawian sites, and, in all settings, the first week of life accounted for most neonatal deaths, ranging from $63 \%$ in Makwanpur (Nepal) and SNEHA (India) to $82 \%$ in MaiKhanda (Malawi).

\section{DISCUSSION}

Using prospective data from seven community-based surveillance systems, we applied an automated method of VA interpretation to provide a uniquely standardised summary and comparison of neonatal mortality across four low-income and middle-income settings. Across all settings, and particularly in rural areas, neonatal mortality rates were unacceptably high and driven by prematurity, birth asphyxia (intrapartum-related deaths) and infections, which were particularly important in the rural Asian sites. The first days of life remain the most risky, with more than one-third of deaths occurring on the first day, half in the first 2 days and three-quarters in the first week.

\section{Gender disparities}

Male gender disadvantage was observed in all study sites, with boys being up to $50 \%$ more likely to die than girls. While our observation could be a consequence of methodological bias, whereby social desirability may lead to an over-reporting for male offspring, a neonatal mortality ratio of males to females of at least 1.2 has been reported previously. ${ }^{37}$ Recent research has also shown that boys are more likely to be born prematurely and have a higher incidence of infections and encephalopathy. ${ }^{39-41}$ With the exception of Dhanusha (Nepal) and PCP (Bangladesh), sex differentials in CSMFs in table 3 suggested more infections in males, although prematurity appeared to act in the opposite direction, and was responsible for a higher proportion of female deaths. Similar findings for late neonatal deaths have been observed in other studies from $\mathrm{Nepal}^{38}$ and India, ${ }^{42}$ and may reflect overarching gender preference or preferential care-seeking as well as interactions between ethnicity, sex and the presence of prior siblings. The extent to which observed sex differentials reflect biological phenomena or are a consequence of modifiable socioeconomic factors demands further investigation.

\section{Differences between countries}

There were important differences in overall mortality and cause distributions between settings. PCP (Bangladesh) and the sites in Malawi and Nepal had similar overall neonatal mortality rates of 25-35 deaths per 1000 live births. However, the proportion of deaths attributed to prematurity, birth asphyxia and infections varied considerably between settings. In the Bangladesh site and both Nepal sites, the burden of the combined infectious causes of death accounted for almost two-fifths of the total mortality burden, whereas in Malawi, the burden ranged from 26\% to $34 \%$, and a greater proportion of prematurity-related mortality was observed. This is perhaps unsurprising given that Malawi is reported to have the highest prematurity rate in the world, at $18 \%$. $^{43}$

\section{Differences within countries}

Ekjut, in rural India, had the highest neonatal mortality rate of 59 deaths per 1000 live births, almost seven times greater than SNEHA in urban Mumbai, reflecting huge subnational variation. Infections and prematurity each accounted for around one-third of neonatal deaths in Ekjut. While infections and prematurity remained in the top three causes in Mumbai, more than one-third of deaths were attributed to asphyxia, making it the leading cause of death. This is in agreement with a neonatal VA study from the same setting, which identified one-third of asphyxia deaths as being associated with obstetric complications. ${ }^{44}$ Observed differences between rural and urban India are likely to reflect documented shifts in cause patterns as overall mortality rates fall, largely driven by decreases in the rates of death from infections, particularly pneumonia. ${ }^{45}$

Within-country differences were also observed in Malawi and Nepal, but are not easily explained by underlying mortality rates. In Malawi, data from MaiMwana suggest that approximately one-third of neonatal deaths were attributable to neonatal pneumonia/sepsis, approximately $8 \%$ greater than the burden identified at the MaiKhanda site, where slightly higher burdens of prematurity and asphyxia were observed. Both MaiMwana and MaiKhanda represent rural Malawian populations, but at slightly different times and within a period of rapid increase in institutional deliveries. ${ }^{36}$ More births occurred in facilities in MaiKhanda than in MaiMwana (table 1). Given that home deliveries are associated with less hygienic practices and higher rates of infectious causes of neonatal death, ${ }^{46}$ the higher proportion of institutional delivery in MaiKhanda may partly explain the differences in cause-specific mortality. A similar 
Table 1 Study populations in descending order of neonatal mortality rate

InterVA disease

prevalence

\section{Study site and area description}

Ekjut—rural India: three rural districts in Jharkhand (Saraikela Kharsawan, West Singhbhum) and Odisha (Keonjhar) $^{22}$

SNEHA—urban India: six municipal wards in Mumbai ${ }^{21}$

Dhanusha—rural Nepal: district excluding Janakpur municipality ${ }^{26}$

Makwanpur-rural Nepal: village development committee areas in Makwanpur district ${ }^{23}$

( $P C P$ - rural Bangladesh: nine unions in three rural districts (Bogra, Faridpur and Moulavibazar) ${ }^{17} 18$

MaiMwana—rural Malawi: Mchinji district ${ }^{19}$

MaiKhanda-rural Malawi: three districts in central region

(Kasungu, Lilongwe and Salima) ${ }^{20}$

*Estimated from available data

PCP, Perinatal Care Project; SNEHA, Society for Nutrition, Education and Health Action; VA, verbal autopsies.

Table 2 Neonatal verbal autopsies (VA) included in the study

\begin{tabular}{|c|c|c|c|c|c|c|c|}
\hline \multirow[b]{2}{*}{ tudy site } & \multirow[b]{2}{*}{$\begin{array}{l}\text { Live births } \\
\text { included }\end{array}$} & \multirow[b]{2}{*}{$\begin{array}{l}\text { Stillbirths included } \\
\text { (proportion of all deaths) }\end{array}$} & \multirow[b]{2}{*}{$\begin{array}{l}\text { Neonatal deaths included } \\
\text { (proportion of all deaths) }\end{array}$} & \multicolumn{3}{|c|}{$\begin{array}{l}\text { Neonatal mortality rate (per } 1000 \text { live } \\
\text { births) }\end{array}$} & \multirow[b]{2}{*}{$\begin{array}{l}\text { Crude neonatal mortality } \\
\text { rate ratio (male: female) }(95 \% \mathrm{Cl})\end{array}$} \\
\hline & & & & Total & Male & Female & \\
\hline kjut, rural India & 8819 & $270(34 \%)$ & $518(66 \%)$ & 59 & 68 & 50 & 1.4 (1.1 to 1.6$)$ \\
\hline NEHA, urban India & 10029 & $80(48 \%)$ & $87(52 \%)$ & 9 & 10 & 7 & $1.3(0.8$ to 2.1$)$ \\
\hline hanusha, Nepal & $15299^{*}$ & $463(47 \%)$ & $528(53 \%)$ & 35 & 39 & 30 & $1.3(1.1$ to 1.5$)$ \\
\hline Makwanpur, Nepal & 6735 & $146(42 \%)$ & $203(58 \%)$ & 30 & 36 & 24 & $1.5(1.1$ to 2.0$)$ \\
\hline $\mathrm{CP}$, rural Bangladesh & 42241 & $1361(51 \%)$ & 1324 (49\%) & 31 & 36 & 27 & 1.3 (1.2 to 1.5$)$ \\
\hline MaiMwana, Malawi & 15258 & $324(46 \%)$ & 382 (54\%) & 25 & 29 & 21 & $1.4(1.1$ to 1.7$)$ \\
\hline MaiKhanda, Malawi & 22 563* & $612(46 \%)$ & $730(54 \%)$ & 32 & 35 & 30 & $1.2(1.0$ to 1.4$)$ \\
\hline
\end{tabular}

*Estimated from available data.

PCP, Perinatal Care Project; SNEHA, Society for Nutrition, Education and Health Action. 


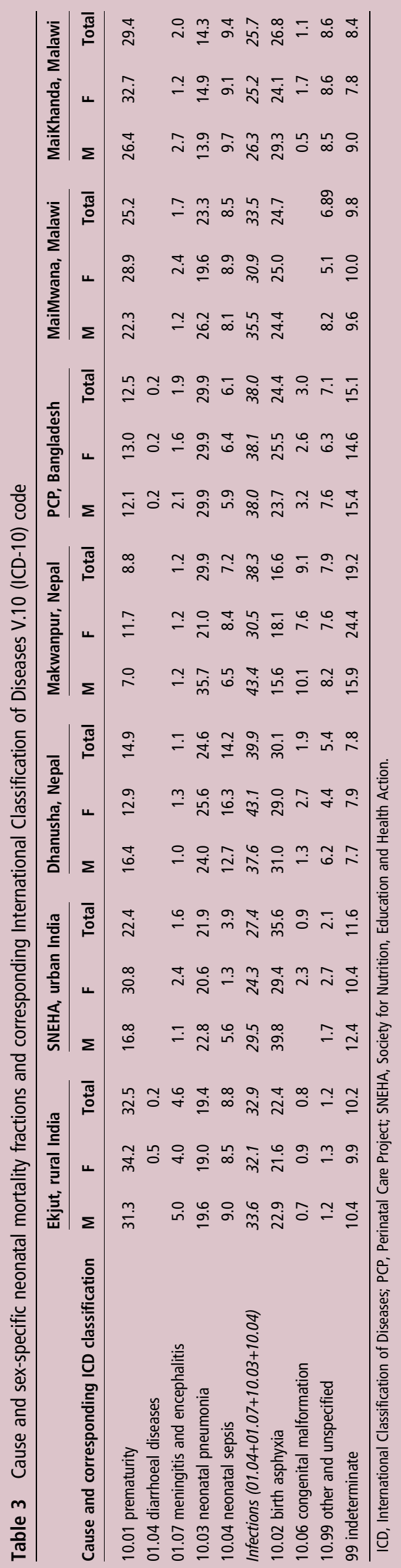

scenario may also explain differences observed between Makwanpur (2\% facility deliveries) and Dhanusha (26\% facility deliveries) in Nepal. However, the different time periods for data collection and contextual differences between the plains of Dhanusha and the hills of Makwanpur may also be important. Finally, the substantial difference in the proportion of deaths with insufficient VA data to attribute a specified cause (categorised as 'indeterminate' and 'other unspecified' in table 3) suggests that observed differences in cause distribution may also relate to underlying differences in VA data completeness.

\section{Study strengths and limitations}

The prospective birth and death surveillance systems, high follow-up rate for completion of VAs, data quality procedures and large sample size are the strengths of the study. There remains a potential bias if certain neonatal deaths were systematically unsuccessfully followed up for VA, but we do not expect this given that the sum of the VA-derived CSMRs closely approximated total mortality rates derived from surveillance in the same areas. ${ }^{17-23}$ The use of InterVA is also a strength in that it allows identification of multiple causes of death per case with quantified degrees of certainty, provides confidence that observed differences are not a consequence of differing methods of assigning causes and has been shown to compare well with available reference standards for cause of death in a range of settings, ${ }^{47}$ including perinatal death classifications. ${ }^{48}$ Indeed, the CSMFs identified by InterVA for Dhanusha and SNEHA were similar to those derived by physician review. ${ }^{44}{ }^{49}$ Cause distributions were also similar between InterVA and physician interpretation of neonatal VA data from Ekjut and PCP (unpublished data). However, physicians at PCP identified asphyxia as the leading cause of death, and Ekjut physicians attributed a substantial number of neonatal deaths to hypothermia, which is not one of the causes included in the 2012 WHO VA standards and, therefore, in InterVA, which may have largely classified such deaths as 'indeterminate'.

Several methodological limitations might have influenced the cause distributions observed. The specific questionnaires used at each site, though similar, were not exactly the same, and the period between neonatal death and VA interview differed. In addition to the potential biases inherent in survey methods and maternal reporting, ${ }^{50}{ }^{51}$ these differences may have influenced VA respondents' ability to recognise, recall and report signs and symptoms. They may also partly explain the differing mean number of non-missing indicators reported per case in each setting, ranging from 12 in MaiKhanda to 17 in Bangladesh. Similarly, it is possible that the processing of common questions into site-specific translations for InterVA may have led to subtle differences in meaning and comprehension. Although there is no empirical evidence on the effect of such factors on VA-derived causes of death, and InterVA was designed with the reality of different VA data collection tools in mind (eg, only affirmative reports of the presence of indicators influence cause probabilities), the possibility that differing cause distributions are driven by differing data capture methods cannot be ruled out.

\section{Comparisons with other studies}

In general, our analysis supports current modelled characterisations of neonatal mortality at an aggregate level. ${ }^{45}$ Our causedistribution findings are also similar in magnitude and rank order to survey results reported elsewhere for comparable settings, ${ }^{44} 495253$ and are broadly similar to descriptions of neonatal mortality from 18 INDEPTH health and demographic surveillance sites using similar methods. ${ }^{54}$ There are some 


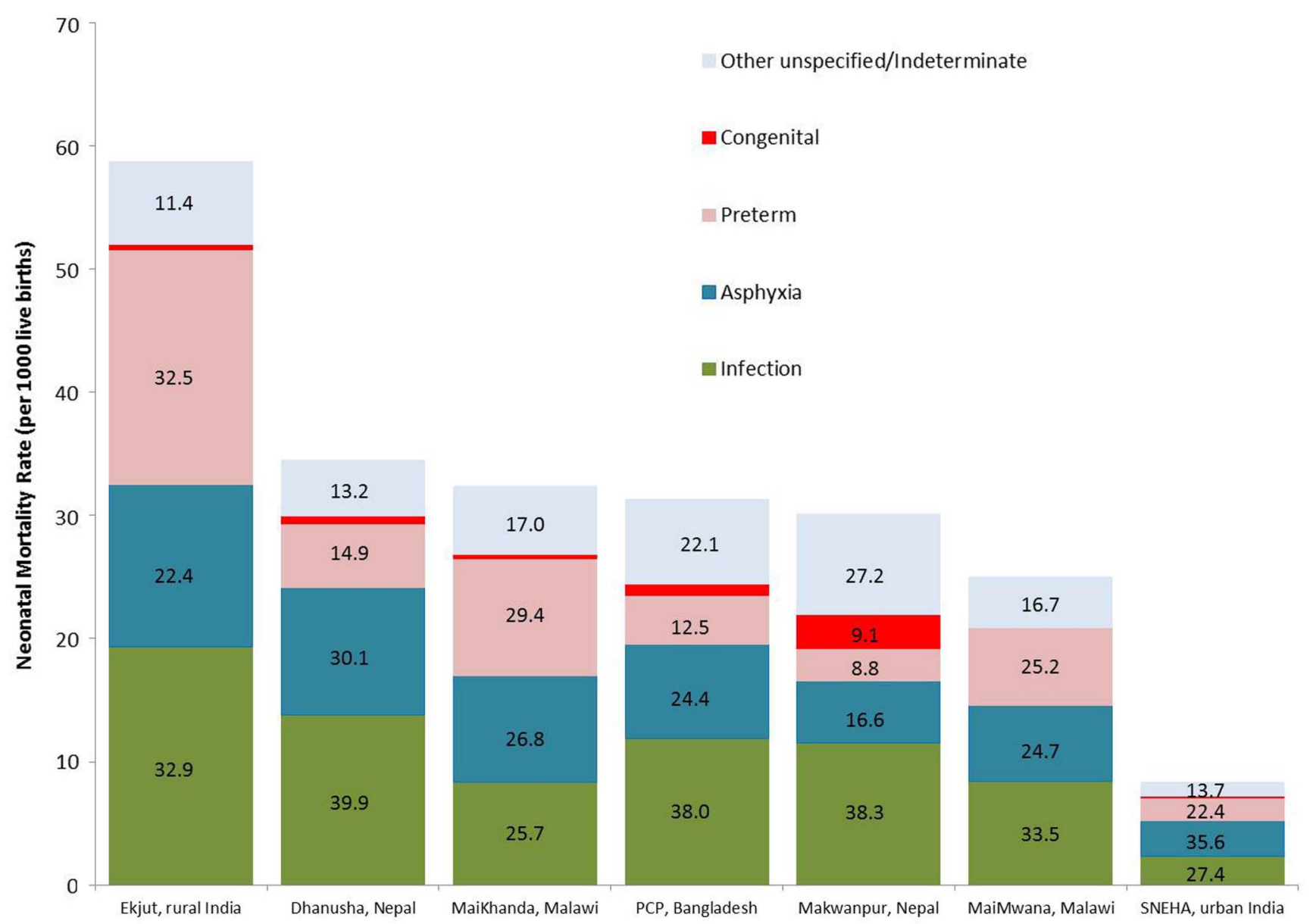

Figure 1 Total and cause-specific neonatal mortality rates by study site ordered left to right by decreasing neonatal mortality rate. Superimposed numbers represent cause-specific mortality fractions (CSMFs) (\%) for each site.

PCP, Perinatal Care Project; SNEHA, Society for Nutrition, Education and Health Action.

notable exceptions. Chowdhury et $a^{55}$ identified birth asphyxia as the leading cause among 365 neonatal deaths in Matlab, rural Bangladesh, accounting for $45 \%$ of the total burden, whereas infection-related causes accounted for less than $20 \%$ of deaths. This contrasts with our findings from Bangladesh, which attributed approximately one-quarter of deaths to asphyxia and a much higher proportion to infections. Conversely, comparisons of our findings with public domain INDEPTH data ${ }^{56}$ for 1619 and 207 neonatal deaths from Bangladesh and India, respectively, identify higher CSMFs of asphyxia and lower proportions of other unspecified/indeterminate causes in our data. While it is possible that such differences reflect varying epidemiology and rates of skilled attendance at birth between different areas, it is also conceivable that they are an artefact of differing VA survey methods. The Matlab study, for example, had a relatively small sample size, and VA interpretation was by a panel of physicians required to reach consensus on a single cause of death for each case. ${ }^{55}$ Neonatal causes of death can be difficult to attribute with certainty, depending on clinical factors and issues of definition; a recent analysis showed a fivefold difference in the proportion of deaths attributed to prematurity depending on definition used. ${ }^{57}$ As such, identifying a single direct cause of death from VA may be unrealistic and masks the uncertainty that may have been evident in the original physicians' consideration of individual cases. Our method, and that used by the INDEPTH group, of calculating population-level CSMFs and splitting deaths between multiple causes is arguably a more epidemiological approach that shifts the focus from individuals to populations. ${ }^{58}$ This fundamental methodological difference may explain the lower overall proportion of asphyxia deaths observed in the INDEPTH datasets as well as ours compared with the study by Chowdhury et al. ${ }^{55}$ Given that both PCP and the INDEPTH data were interpreted using InterVA V.4.02 however, differences between these datasets must be due to other reasons, possibly underlying differences in the time and location of the surveys, or differences in VA data capture.

\section{Programmatic implications}

At all study sites, stillbirths accounted for a large proportion of deaths in the extended perinatal period. The estimates in our data ranged from $34 \%$ in Ekjut, India, to $51 \%$ in PCP, Bangladesh. All methods used to distinguish stillbirths from early neonatal deaths are open to misclassification bias, influenced by sociocultural factors and limited assessment of vital signs or attempts at resuscitation in apparently lifeless newborns, particularly after home birth. ${ }^{59}$ Misclassification bias notwithstanding, the burden of stillbirths remains high and is likely to reflect inadequacies in skilled attendance at birth and the availability of emergency obstetric care and caesarean sections.

The neonatal cause-of-death distributions described here have important programmatic implications. High levels of infection and prematurity-related deaths imply that essentials of hygienic delivery and newborn care, including thermal care, remain priorities. Preventative strategies that target maternal health, 


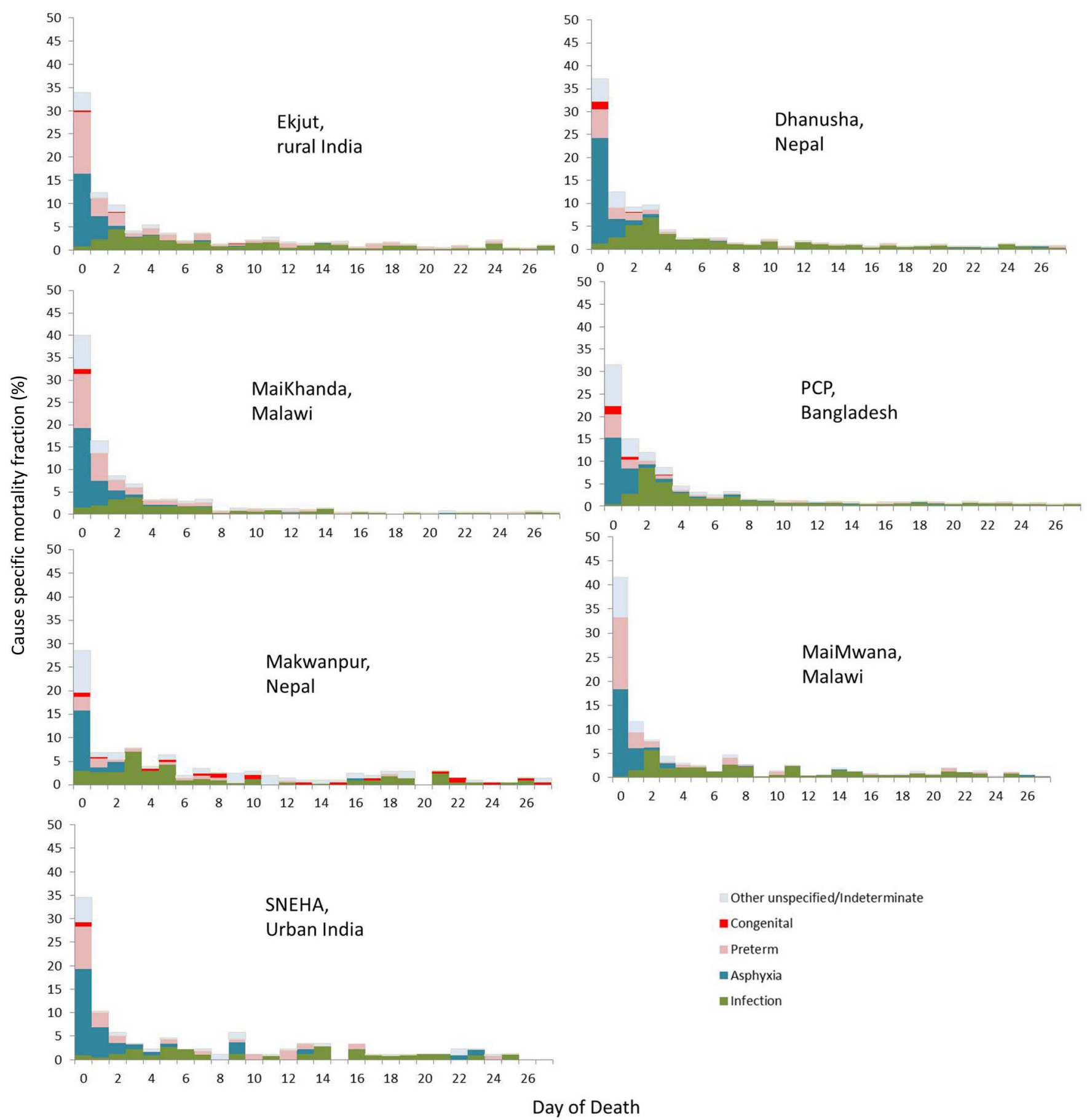

Figure 2 Cause-specific mortality fractions (CSMFs) by day of death and study site ordered left to right by decreasing neonatal mortality rate. PCP, Perinatal Care Project; SNEHA, Society for Nutrition, Education and Health Action.

recognising the importance of nutrition and vector-borne diseases such as malaria, a major cause of anaemia in malaria endemic regions, must also be prioritised. At the same time, the major burden of asphyxia mortality demands a focus on the intrapartum period, with improved capacity within facilities to manage obstetric complications and awareness of the importance of skilled attendance at birth among communities and expectant mothers. Given the high rates of home births, intervention strategies must think beyond the biomedical aspects of mortality described by VA results and consider the distal, social determinants of mortality and the three delays model.$^{60}$ In this regard, there is a clear need to focus on community interventions for newborn health that also encompass the health of the mother and the continuum of preconception, pregnancy and the intrapartum and postpartum periods, and which promote hygienic practices, thermal care, the recognition of danger signs and appropriate care-seeking. There is a growing body of evidence suggesting that cost-effective community interventions achieving impact on neonatal mortality should be scaled up as a matter of urgency. ${ }^{24} 6162$

\section{CONCLUSION}

The application of simple mortality surveillance and VA methods in localised, low-income and middle-income settings 
reveals important regional and subnational variation in the epidemiology of neonatal mortality that may be masked by aggregate or modelled estimates. Although population health survey and VA approaches are likely to be imperfect compared with complete vital registration and medical cause of death certification, methodological advances enable the generation of timely, low-cost and consistent cause-specific mortality estimates necessary to direct local policy in settings with little or no existing data. The wider application of these methods beyond research settings to better inform and strengthen health systems is a global priority. However, it is not for lack of data that the burden of neonatal mortality remains so high; efforts must focus on implementing proven interventions to protect the three million newborns who die every year.

\author{
Author affiliations \\ ${ }^{1}$ University College London, Institute for Global Health, London, UK \\ ${ }^{2}$ Diabetic Association of Bangladesh, Perinatal Care Project, Dhaka, Bangladesh \\ ${ }^{3}$ Society for Nutrition, Education and Health Action, Mumbai, India \\ ${ }^{4}$ Parent and Child Health Initiative, Lilongwe, Malawi \\ ${ }^{5}$ Mother and Infant Research Activities, Kathmandu, Nepal \\ ${ }^{6}$ Ministry of Health, Government of Malawi, Lilongwe, Malawi \\ ${ }^{7}$ Ekjut, Chakradharpur, India \\ ${ }^{8}$ MaiMwana Project, Mchinji, Malawi
}

\section{Twitter Follow Edward Fottrell at @fottrell}

Acknowledgements The authors gratefully acknowledge fieldwork, coordination and management teams in the Perinatal Care Project, Bangladesh; the Society for Nutrition, Education and Health Action, India; the Parent and Child Health Initiative, Malawi; Mother and Infant Research Activities, Nepal; Ekjut, India; and the MaiMwana Project, Malawi, for their crucial role in data acquisition.

Contributors EF conceived the study, carried out analysis and wrote the first draft of the manuscript. DO, GA, UB, SD and NSM led data capture activities and data processing in Mumbai. EF, KA and JB led data capture activities and data processing in Bangladesh. $A B, T C, C K, C M, B N, T P$ and $B S$ led data capture activities and data processing and interpretation in Malawi. DM, SM, JM, NSa, AS, BPS, KMT and JB led data capture activities and data processing and interpretation in Nepal. NN and AP led data capture activities and data processing in Ekjut, rural India. MN and NSe contributed to data pooling and processing. AC established the international collaboration between all partners and helped to design the analysis plan. EF, AP, DO, TC, MN and NN provided technical oversight to analysis and interpretation of data. All authors contributed to context-specific interpretation of the results and revisions of the manuscript.

Funding This work was supported by a Wellcome Trust Strategic Award (085417ma/Z/08/Z).

\section{Competing interests None declared.}

Ethics approval The University College London or the Great Ormond Street Hospital Research Ethics Committee, UK; the Ethical Review Committee of the Diabetic Association of Bangladesh; the Nepal Health Research Council (with a Memorandum of Understanding with the Government of Nepal Ministry of Health); the Independent Ethics Committee for Research on Human Subjects (Mumbai), India; the Malawi National Health Sciences Research Committee.

Provenance and peer review Not commissioned; externally peer reviewed.

Data sharing statement We will make an anonymised version of the dataset used for the analyses in this article available to researchers upon completion of a simple data request form, which can be obtained from Dr Audrey Prost (Audrey. prost@ucl.ac.uk).

Open Access This is an Open Access article distributed in accordance with the terms of the Creative Commons Attribution (CC BY 4.0) license, which permits others to distribute, remix, adapt and build upon this work, for commercial use, provided the original work is properly cited. See: http://creativecommons.org/ licenses/by/4.0/

\section{REFERENCES}

1 Lozano $\mathrm{R}$, Wang $\mathrm{H}$, Foreman $\mathrm{KJ}$, et al. Progress towards Millennium Development Goals 4 and 5 on maternal and child mortality: an updated systematic analysis. Lancet 2011:378:1139-65.

2 Kinney MV, Kerber KJ, Black RE, et al. Sub-Saharan Africa's Mothers, Newborns, and Children: Where and Why Do They Die? PLoS Med 2010;7:e1000294.
3 Black RE, Cousens S, Johnson $\mathrm{HL}$, et al. Global, regional, and national causes of child mortality in 2008: a systematic analysis. Lancet 2010;375:1969-87.

4 Lawn J, Kinney M, Black R, et al. Newborn survival: a multi-country analysis of a decade of change. Health Policy Plan 2012;27:iii6-iii28.

5 Oestergaard MZ, Inoue M, Yoshida S, et al. Neonatal mortality levels for 193 countries in 2009 with trends since 1990: a systematic analysis of progress, projections and priorities. PLoS Med 2011;8:e1001080.

6 Jamison DT, Summers LH, Alleyne G, et al. Global health 2035: a world converging within a generation. Lancet 2013;382:1898-955

7 WHO. Every Newborn: a draft actionplan to end preventable deaths. Geneva: World Health Organization, 2014

8 Lawn JE, Blencowe $\mathrm{H}, \mathrm{Oza} \mathrm{S}$, et al. Progress, priorities, and potential beyond survival. Lancet 2014:384:189-205.

9 Lozano R, Naghavi M, Foreman K, et al. Global and regional mortality from 235 causes of death for 20 age groups in 1990 and 2010: a systematic analysis for the Global Burden of Disease Study 2010. Lancet 2012;380:2095-128.

10 Byass $\mathrm{P}$, de Courten $\mathrm{M}$, Graham WJ, et al. Reflections on the Global Burden of Disease 2010 Estimates. PLoS Med 2013;10:e1001477.

11 Mathers CD, Boerma T. Mortality measurement matters: improving data collection and estimation methods for child and Adult Mortality. PLoS Med 2010;7:e1000265

12 Chan M. From new estimates to better data. Lancet 2012;380:2054.

13 WHO. Verbal autopsy standards: the 2012 WHO verbal autopsy instruments (release candidate 1). Geneva: WHO, 2012.

14 Byass P. The UN needs joined-up thinking on vital registration. Lancet 2012;380:1643.

15 WHO. Commission on information and accountability for Women's and Children's Health: keeping promises, measuring results. Geneva: World Health Organization, 2011.

16 WHO. Every Woman, Every Child: strengthening equity and dignity through health the second report of the independent Expert Review Group (iERG) on information and accountability for Women's and Children's health. Geneva: World Health Organization, 2013.

17 Azad K, Barnett S, Banerjee B, et al. Effect of scaling up women's groups on birth outcomes in three rural districts in Bangladesh: a cluster-randomised controlled trial. Lancet 2010;375:1193-202.

18 Fottrell E, Azad K, Kuddus A, et al. The effect of increased coverage of participatory women's groups on neonatal mortality in Bangladesh: a cluster randomized trial. JAMA Pediatr 2013;167:816-25.

19 Lewycka S, Mwansambo C, Rosato M, et al. Effect of women's groups and volunteer peer counsellors on mortality, morbidity and health behaviours among mothers and children in rural Malawi: the MaiMwana cluster randomised controlled trial. Lancet 2013:381:1721-35.

20 Colbourn T, Nambiar B, Bondo A, et al. Effects of quality improvement in health facilities and community mobilisation through women's groups on maternal, neonatal and perinatal mortality in three districts of Malawi: MaiKhanda, a cluster randomised controlled effectiveness trial. Int Health 2013;5:180-95.

21 More NS, Bapat U, Das S, et al. Community Mobilization in Mumbai Slums to Improve Perinatal Care and Outcomes: A Cluster Randomized Controlled Trial. PLoS Med 2012;9:e1001257.

22 Tripathy P, Nair N, Barnett $S$, et al. Effect of a participatory intervention with women's groups on birth outcomes and maternal depression in Jharkhand and Orissa, India: a cluster-randomised controlled trial. Lancet 2010;375:1182-92.

23 Manandhar DS, Osrin D, Shrestha BP, et al. Effect of a participatory intervention with women's groups on birth outcomes in Nepal: cluster-randomised controlled trial. Lancet 2004;364:970-9

24 Prost A, Colbourn T, Seward N, et al. Women's groups practising participatory learning and action to improve maternal and newborn health in low-resource settings: a systematic review and meta-analysis. Lancet 2013;381:1736-46.

25 Azad $K$. The impact of TBA resuscitation training on early neonatal mortality in Bangladesh: results and implications. Presentation from Towards 4+5 conference; 25-26 May 2010. London, UK: DfID, 2010.

26 Shrestha BP, Bhandari B, Manandhar DS, et al. Community interventions to reduce child mortality in Dhanusha, Nepal: study protocol for a cluster randomized controlled trial. Trials 2011;12:136.

27 Osrin D, Manandhar A, Shrestha A, et al. Design of a surveillance system for pregnancy and its outcomes in rural Nepal. J Nepal Med Assoc 2003;41:411-22.

28 Barnett $S$, Nair $N$, Tripathy $P$, et al. A prospective key informant surveillance system to measure maternal mortality - findings from indigenous populations in Jharkhand and Orissa, India. BMC Pregnancy \& Childbirth 2008;8:6.

29 Lewycka S, Mwansambo C, Kazembe $P$, et al. A cluster randomised controlled trial of the community effectiveness of two interventions in rural Malawi to improve health care and to reduce maternal, newborn and infant mortality. Trials 2010;11:88.

30 Colbourn T, Nambiar B, Costello A. MaiKhanda_Final evaluation report The impact of quality improvement at health facilities and community mobilisation by women's groups on birth outcomes: an effectiveness study in three districts of Malawi. London: The Health Foundation, 2013.

31 Fottrell E, Byass P. Verbal autopsy: methods in transition. Epidemio/ Rev 2010;32:38-55. 
32 Fottrell E, Byass $P$, Ouedraogo $T$, et al. Revealing the burden of maternal mortality: a probabilistic model for determining pregnancy-related causes of death from verba autopsies. Popul Health Metr 2007;5:1.

33 Byass P, Chandramohan D, D'Ambruoso L, et al. Strengthening standardized interpretation of verbal autopsy data: the new InterVA-4 tool. Global Health Action 2012;5:1-8.

34 InterVA. User Guide.

35 Fottrell E, Kahn K, Tollman SM, et al. Probabilistic methods for verbal autopsy interpretation: InterVA robustness in relation to variations in a priori probabilities. PLOS ONE 2011;6:e27200

36 National Statistical Office (NSO) and ICF Macro. Malawi Demographic and Health Survey 2010. Zomba, Malawi, and Calverton, Maryland, USA: NSO and ICF Macro, 2011.

37 Lawn J, Kerber K, Enweronu-Laryea C, et al. 3.6 million neonatal deaths-what is progressing and what is not? Semin Perinatol 2010;34:371-86.

38 Rosenstock S, Katz J, Mullany L, et al. Sex differences in neonatal mortality in Sarlahi, Nepal: the role of biology and environment. J Epidemiol Community Health 2013;67:986-91.

39 Lawn J, Blencowe H, Darmstadt G, et al. Beyond newborn survival: the world you are born into determines your risk of disability-free survival. Pediatr Res 2013:74:1-3.

40 Lee $\mathrm{A}$, Kozuki N, Blencowe $\mathrm{H}$, et al. Intrapartum-related neonatal encephalopathy incidence and impairment at regional and global levels for 2010 with trends from 1990. Pediatr Res 2013:74(Suppl 1):50-72.

41 Seale A, Blencowe $H$, Zaidi A, et al. Neonatal severe bacterial infection impairment estimates in South Asia, sub-Saharan Africa, and Latin America for 2010. Pediatr Res 2013;74(Suppl 1):73-85.

42 Krishnan $\mathrm{A}, \mathrm{Ng} \mathrm{N}$, Kapoor $\mathrm{S}$, et al. Temporal trends and gender differentials in causes of childhood deaths at Ballabgarh, India-need for revisiting child survival strategies. BMC Public Health 2012;12:555.

43 March of Dimes, PMNCH, Save the Children, WHO. Born Too Soon: The Global Action Report on Preterm Birth. In Howson CP, Kinney MV, Lawn JE, eds. Geneva: World Health Organization, 2012.

44 Bapat U, Alcock G, More N, et al. Stillbirths and newborn deaths in slum settlements in Mumbai, India: a prospective verbal autopsy study. BMC Pregnancy Childbirth 2012;12:39.

45 Bhutta ZA, Black RE. Global maternal, newborn and child health-so near yet so far. N Engl J Med 2013;639:2226-35.

46 Seward N, Osrin D, Li L, et al. Association between clean delivery kit use, clean delivery practices, and neonatal survival: pooled analysis of data from three sites in South Asia. PLoS Med 2012;9:e1001180.

47 Byass $P$, Herbst A, Fottrell $E$, et al. Comparing verbal autopsy cause of death findings as determined by physician coding and probabilistic modelling: a public health analysis of 54,000 deaths in Africa and Asia. J Glob Health 2015;5:010402.
48 Vergnano S, Fottrell E, Osrin D, et al. Adaptation of a probabilistic method (InterVA) of verbal autopsy to improve the interpretation of cause of stillbirth and neonatal death in Malawi, Nepal and Zimbabwe. Popul Health Metr 2011;9:48.

49 Manandhar S, Ojha A, Manandhar D, et al. Causes of stillbirths and neonatal deaths in Dhanusha district, Nepal: a verbal autopsy study. Kathmandu Univ Med J (KUMJ) 2010;8:62-72

50 Yoder P, Rosato M, Mahmud R, et al. Women's recall of delivery and neonatal care: a study of terms, concepts and survey questions. Calverton, Maryland, USA: ICF Macro, 2010.

51 Snow RW, Basto de Azevedo I, Forster D, et al. Maternal recall of symptoms associated with childhood deaths in rural east Africa. Int J Epidemiol 1993;22:677-83.

52 Baqui $\mathrm{AH}$, Darmstadt GL, Williams EK, et al. Rates, timing and causes of neonatal deaths in rural India: implications for neonatal health programmes. Bull World Health Organ 2006;84:706-13.

53 Khanal S, Gc V, Dawson P, et al. Verbal autopsy to ascertain causes of neonatal deaths in a community setting: a study from Morang, Nepal. JNMA J Nepal Med Assoc 2011:51:21-7.

54 Streatfield P, Khan W, Bhuiya A, et al. Cause-specific childhood mortality in Africa and Asia: evidence from INDEPTH health and demographic surveillance systems. Glob Health Action 2014; 7:25363.

55 Chowdhury $\mathrm{H}$, Thompson S, Ali M, et al. Causes of neonatal deaths in a rural subdistrict of Bangladesh: implications for intervention. J Health Popul Nutr 2010;28:375-82.

56 INDPETH. Africa, Asia, Oceania-INDEPTH Network Cause-Specific MortalityRelease 2014. 2014. http://www.indepth-ishare.org/index.php/catalog/48

57 Byass P. Usefulness of the Population Health Metrics Research Consortium gold standard verbal autopsy data for general verbal autopsy methods. BMC Med 2014;12:23.

58 Byass $P$, Kahn $K$, Fottrell $E$, et al. Moving from data on deaths to public health policy in Agincourt, South Africa: approaches to analysing and understanding verbal autopsy findings. PLoS Med 2010;7:e1000325.

59 Fron J, Gordin S, Abdel-Aleem H, et al. Making stillbirths count, making numbers talk —issues in data collection for stillbirths. BMC Pregnancy Childbirth 2009;9:58.

60 Upadhyay R, Krishnan A, Rai S, et al. Need to focus beyond the medical causes: a systematic review of the social factors affecting neonatal deaths. Paediatr Perinatal Epidemiol 2014;28:127-37.

61 Kirkwood B, Manu A, ten Asbroek A, et al. Effect of the Newhints home-visits intervention on neonatal mortality rate and care practices in Ghana: a cluster randomised controlled trial. Lancet 2013:381:2184-92.

62 Lawn J, Mwansa-Kambafwile J, Horta B, et al. 'Kangaroo mother care' to prevent neonatal deaths due to preterm birth complications. Int J Epidemiol 2010;39(suppl 1):i144-i54.

63 Clarke K, Saville N, Shrestha B, et al. Predictors of psychological distress among postnatal mothers in rural Nepal: A cross-sectional community-based study. J Affect Disord 2014;156:76-86. 